\title{
Search for the $\eta$-mesic bound states with the WASA-at-COSY detector
}

\author{
Magdalena Skurzok ${ }^{1, *}$ \\ for the WASA-at-COSY Collaboration \\ ${ }^{1}$ M. Smoluchowski Institute of Physics, Jagiellonian University, Cracow, Poland
}

\begin{abstract}
The experiments dedicated to the search for $\eta$-mesic Helium were performed with high statistics using WASA detection setups installed at the COSY accelerator in the Research Center Juelich. The search for the $\eta$-mesic bound states is conducted via the measurement of the excitation function for selected decay channels of the He- $\eta$ systems using unique ramped beam technique. This report presents recent status of the data analysis.
\end{abstract}

\section{Introduction}

The mesic nuclei issue currently attracts much interest in nuclear and hadronic physics, both from experimental [1-7] and theoretical points of view [8-27]. Although, this exotic nuclear matter consisting of a nucleus bound via the strong interaction with a neutral meson $\left(\eta, \eta^{\prime}\right.$, $K$ or $\omega$ ) has been predicted over thirty years ago [28, 29], its existence remains unconfirmed experimentally. One of the most promising candidates for the creation of the mesic nucleus is the $\eta$ meson whose interaction with nucleons is stronger than other mesons [30, 31]. Current investigations of hadron- and photon-induced production of the $\eta$ meson deliver a wide range of $\eta N$ scattering length values $\left(a_{\eta N}\right)$ indicating the $\eta$-nucleon interaction to be strong enough to create light $\eta$-mesic nuclei [9-12,32-34]. However, despite many experiments performed, there is still no clear evidence of their existence [35-42]. The interested reader can find recent reviews on the $\eta$ mesic bound state searches in Refs [6, 7, 16, 18, 30, 45-49].

Some of the promising experiments related to $\eta$-mesic Helium nuclei have been performed with the COSY facility [50]. COSY-11 group carried out measurements to search for ${ }^{3} \mathrm{He}-\eta$ bound states in $d p \rightarrow p p p \pi^{-}$and $d p \rightarrow{ }^{3} \mathrm{He} \pi^{0}$ reactions. Excitation functions determined in the vicinity of the production threshold allowed to establish the upper limits of the total cross section to about $270 \mathrm{nb}$ and $70 \mathrm{nb}$, respectively [51, 52]. The search for ${ }^{4} \mathrm{He}-\eta$ and ${ }^{3} \mathrm{He}-\eta$ mesic nuclei has been recently performed by WASA-at-COSY Collaboration in $d d$ and $p d$ collisions, respectively. This paper focuses on the results obtained for the search for $\eta$-mesic ${ }^{4} \mathrm{He}$ in $d d \rightarrow{ }^{3} \mathrm{He} n \pi^{0}$ and $d d \rightarrow{ }^{3} \mathrm{He} p \pi^{-}$processes [1-4]. The analysis related to ${ }^{3} \mathrm{He}-\eta$ bound state is in progress [53].

\section{Search for the $\eta$-mesic ${ }^{4} \mathrm{He}$}

\footnotetext{
*e-mail: magdalena.skurzok@uj.edu.pl
} 
Measurements with the WASA-at-COSY detection setup dedicated to search for $\eta$-mesic ${ }^{4} \mathrm{He}$ nuclei were carried out twice (in 2008 and 2010) using the unique ramped beam technique which allows for the beam momentum to be changed slowly and continuously around the $\eta$ production threshold in each of the acceleration cycles [1, 4, 47, 49]. The advantage of this technique is the reduction of systematic uncertainties with respect to separate runs at fixed beam energies $[4,38]$.

The search for ${ }^{4} \mathrm{He}-\eta$ bound states was carried out by studying the excitation functions for $d d \rightarrow{ }^{3} \mathrm{He} p \pi^{-}[1-4]$ and $d d \rightarrow{ }^{3} \mathrm{He} n \pi^{0}[1-3]$ reactions near the ${ }^{4} \mathrm{He} \eta$ production threshold. A detailed description of the data analysis and simulations leading to determination of the excitation functions is presented in the Refs [1,4]. Since the obtained excitation functions do not reveal any direct narrow structure below the $\eta$ production threshold, which could be considered as a signature of the bound state, the upper limit of the total cross section for the $\eta$-mesic ${ }^{4} \mathrm{He}$ formation was determined at the $90 \%$ confidence level. For this purpose the excitation curves were fitted simultaneously using the Breit-Wigner function (signal) with a fixed binding energy and width combined with a second order polynomial (background) taking into account the isospin relation between $n \pi^{0}$ and $p \pi^{-}$pairs.

In case of the $d d \rightarrow\left({ }^{4} \mathrm{He}-\eta\right)_{\text {bound }} \rightarrow{ }^{3} \mathrm{He} n \pi^{0}$ process, the upper limit was for the first time obtained experimentally and varies in the range from 2.5 to $3.5 \mathrm{nb}$. For the $d d \rightarrow\left({ }^{4} \mathrm{He}-\right.$ $\eta)_{\text {bound }} \rightarrow{ }^{3} \mathrm{He} p \pi^{-}$reaction we achieved a sensitivity of the cross section of about $6 \mathrm{nb}$ [1] which is about four times better in comparison with the result obtained in the previous experiment [4]. The obtained upper limits as a function of the bound state width are presented for both of the studied reactions in Fig. 1.
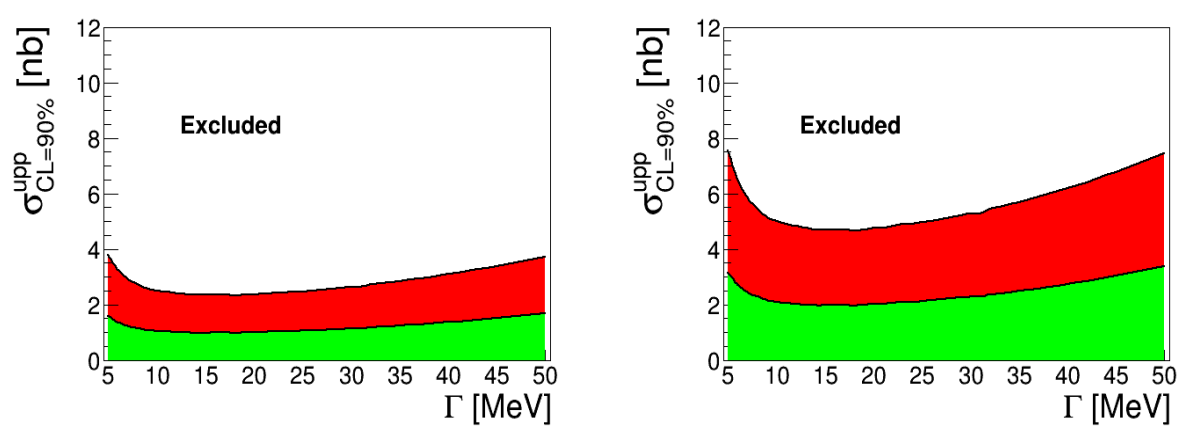

Figure 1. Upper limit of the total cross-section for $d d \rightarrow\left({ }^{4} \mathrm{He}-\eta\right)_{\text {bound }} \rightarrow{ }^{3} \mathrm{He} n \pi^{0}$ (left panel) and $d d \rightarrow\left({ }^{4} \mathrm{He}-\eta\right)_{\text {bound }} \rightarrow{ }^{3} \mathrm{He} p \pi^{-}$(right panel) reaction as a function of the width of the bound state. The binding energy was fixed to $30 \mathrm{MeV}$. The upper limit was determined via the simultaneous fit for both channels. The green area denotes the systematic uncertainties. The figures are adopted from [1].

\subsection{Constraining the optical potential}

Previously, the data analyses were carried out assuming that the signal from the bound state is described by a Breit-Wigner shape with fixed binding energy and width [1, 4], since there were no theoretical predictions for the $d d \rightarrow\left({ }^{4} \mathrm{He}-\eta\right)_{\text {bound }} \rightarrow{ }^{3} \mathrm{He} N \pi$ reactions cross sections below the $\eta$ production threshold. However, at present, a theoretical description of the cross sections in the excess energy range relevant to the $\eta$-mesic nuclear search was proposed in Ref. [8]. The authors used a phenomenological approach with an optical potential for the $\eta-{ }^{4} \mathrm{He}$ interaction and determine the total cross sections for a broad range of real $\left(V_{0}\right)$ and imaginary $\left(W_{0}\right)$ parameters. 
Fitting the theoretical spectra convoluted with the experimental resolution of the excess energy (example Fig. 2) to experimental data collected by WASA-at-COSY [1] brought the upper limit of the total cross section $(\mathrm{CL}=90 \%)$ for creation of $\eta$-mesic nuclei via the $d d \rightarrow{ }^{3} \mathrm{He} N \pi$ process varying from about $5.2 \mathrm{nb}$ to about $7.5 \mathrm{nb}$.

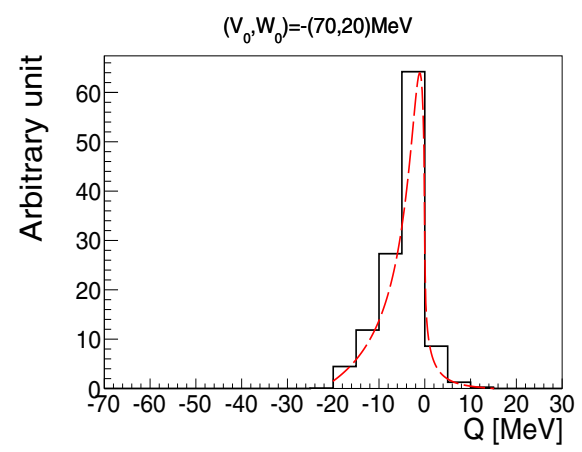

Figure 2. Cross section of the $d d \rightarrow\left({ }^{4} \mathrm{He}-\eta\right)_{\text {bound }} \rightarrow{ }^{3} \mathrm{He} N \pi$ reaction for the formation of the ${ }^{4} \mathrm{He}-\eta$ bound system calculated for $\eta-{ }^{4} \mathrm{He}$ optical potential parameters $\left(V_{0}, W_{0}\right)=-(70,20) \mathrm{MeV}$, plotted as a function of the excess energy $\mathrm{Q}$. The red dashed line shows the theoretical spectrum while the black solid line shows the convoluted spectrum. Figure is adopted from Ref. [2].

A comparison of the determined upper limits with the cross sections obtained in Ref. [8] made it possible to put a constraint on the $\eta{ }^{4} \mathrm{He}$ optical potential parameters. As it is presented in Fig. 3, only extremely narrow and loosely bound states are allowed within the model [8]. A detailed description of performed studies interested reader can find in Ref. [2].

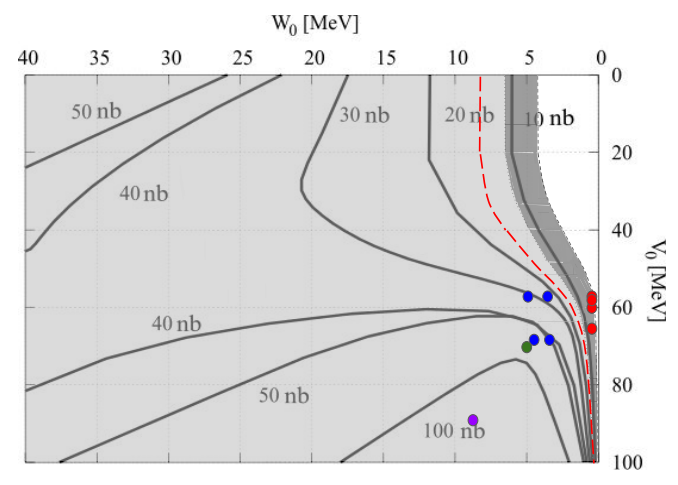

Figure 3. Contour plot of the theoretically determined conversion cross section in the $V_{0}-W_{0}$ plane [8]. The light shaded area shows the region excluded by our analysis, while the dark shaded area denotes the systematic uncertainty of the $\sigma_{u p p}^{C L=90 \%}$. The red line extends the allowed region based on a new estimate of errors (see text for details). Dots correspond to the optical potential parameters corresponding to the predicted $\eta$-mesic ${ }^{4} \mathrm{He}$ states. Figure is adopted from Ref. [2].

\section{Summary and perspectives}


Experiments dedicated to the search for $\eta$-mesic ${ }^{4} \mathrm{He}$ in $d d \rightarrow{ }^{3} \mathrm{He} n \pi^{0}$ and $d d \rightarrow{ }^{3} \mathrm{He} p \pi^{-}$ reactions performed with the WASA-at-COSY detection setup did not enable the observation of the resonance structure related to the bound state. However, the upper limits of the total cross sections for $\eta$-mesic nuclei formation and decay were determined to be of the order of a few nb $[1,4]$. Moreover, a comparison between the phenomenological model proposed in Ref. [8] and experimental data allowed, for the first time, to constrain the range of the $\eta-{ }^{4} \mathrm{He}$ optical potential parameters [2].

Recently, the WASA-at-COSY Collaboration has performed a promising experiment dedicated to the search for $\eta$-mesic ${ }^{3} \mathrm{He}$ in three different mechanisms: (i) absorption of the $\eta$ meson by one of the nucleons, which subsequently decays into $N^{*}-\pi$ pair e.g.: $p d \rightarrow\left({ }^{3} \mathrm{He}-\eta\right)_{\text {bound }} \rightarrow p p p \pi^{-}$, (ii) $\eta$-meson decay while it is still "orbiting" around a nucleus e.g.: $p d \rightarrow\left({ }^{3} \mathrm{He}-\eta\right)_{\text {bound }} \rightarrow{ }^{3} \mathrm{He} 2 \gamma$ reactions and (iii) $\eta$ meson absorption by few nucleons e.g.: $p d \rightarrow\left({ }^{3} \mathrm{He}-\eta\right)_{\text {bound }} \rightarrow p p n$. The measurement with a high average luminosity $\left(3 \cdot 10^{30} \mathrm{~cm}^{-2} \mathrm{~s}^{-1}\right)$ allowed the collection of the largest data sample, in the world available up to now, for ${ }^{3} \mathrm{He}-\eta$ [53-55]. The analysis is still in progress and the estimated upper limit value for $p d \rightarrow{ }^{3} \mathrm{He} 2 \gamma$ and $p d \rightarrow{ }^{3} \mathrm{He} 6 \gamma$ channels is on the level of a few nanobarns.

We acknowledge the support from the Polish National Science Center through grants no. 2013/11/N/ST2/04152 and 2016/23/B/ST2/00784.

\section{References}

[1] P. Adlarson et al., Nucl. Phys. A 959, 102-115 (2017)

[2] M. Skurzok et al., Phys. Lett. B 772, 663 (2018)

[3] M. Skurzok, P. Moskal, W. Krzemien, Prog. Part. Nucl. Phys. 67, 445 (2012)

[4] P. Adlarson et al., Phys. Rev. C 87, 035204 (2013)

[5] Y. K. Tanaka et al., Phys. Rev. Lett. 117, 202501 (2016)

[6] H. Machner, J. Phys. G 42, 043001 (2015)

[7] V. Metag, M. Nanova and E. Ya. Paryev, Prog. Part. Nucl. Phys. 97, 199 (2017)

[8] N. Ikeno et al., Eur. Phys. J. A 53 no.10, 194 (2017)

[9] J. J. Xie et al., Phys. Rev. C 95, 015202 (2017)

[10] A. Fix et al., Phys. Lett. B 772, 663 (2017)

[11] N. Barnea, B. Bazak, E. Friedman and A. Gal, Phys. Lett. B 771, 297 (2017)

[12] N. Barnea, E. Friedman and A. Gal, Nucl. Phys. A 968, 35 (2017)

[13] N. Barnea, E. Friedman and A. Gal, Phys. Lett. B 747, 345 (2015)

[14] E. Friedman, A. Gal, J. Mares, Phys. Lett. B 725, 334 (2013)

[15] N. G. Kelkar, Eur. Phys. J. A 52, 309 (2016)

[16] N. G. Kelkar et al., Rept. Progr. Phys. 76, 066301 (2013)

[17] N. G. Kelkar, Acta Phys. Pol. B 46, 1131 (2015)

[18] C. Wilkin, Acta Phys. Pol. B 47, 249 (2016)

[19] C. Wilkin, Phys. Lett. B 654, 92 (2007)

[20] S. D. Bass, A. W. Thomas, Phys. Lett. B 634, 368 (2006)

[21] S. D. Bass, A. W. Thomas, Acta Phys. Polon. B 41, 2239 (2010)

[22] S. Hirenzaki and H. Nagahiro, Acta Phys. Polon. B 45, 619 (2014)

[23] H. Nagahiro, D. Jido and S. Hirenzaki, Phys. Rev. C 80, 025205 (2009)

[24] H. Nagahiro et al., Phys. Rev. C 87, 045201 (2013)

[25] S. Hirenzaki et al., Acta Phys. Polon. B 41, 2211 (2010)

[26] S. Wycech, W. Krzemien, Acta Phys. Pol. B 45, 745 (2014) 
[27] J. Niskanen, Phys. Rev. C 92, 055205 (2015)

[28] Q. Haider, L. C. Liu, Phys. Lett. B 172, 257 (1986)

[29] R. S. Bhalerao and L. C. Liu, Phys. Rev. Lett. 54, 865 (1985)

[30] P. Moskal, Few Body Syst. 50, 667 (2014)

[31] P. Moskal et al., Phys. Lett. B 482, 356 (2000)

[32] A. M. Green, J. A. Niskanen, S. Wycech, Phys. Rev. C 54, 1970 (1996)

[33] C. Wilkin, Phys. Rev. C 47, 938 (1993)

[34] S. Wycech, A. M. Green and J. A. Niskanen, Phys. Rev. C 52, 544 (1995)

[35] J. Berger et al., Phys. Rev. Lett. 61, 919 (1988)

[36] B. Mayer et al., Phys. Rev. C 53, 2068 (1996)

[37] G. A. Sokol, L. N. Pavlyuchenko, arXiv:nucl-ex/0111020 (2001)

[38] J. Smyrski et al., Phys. Lett. B 649, 258 (2007)

[39] T. Mersmann et al., Phys. Rev. Lett. 98, 242301 (2007)

[40] A. Budzanowski, et al., Phys. Rev. C 79, 012201(R) (2009)

[41] M. Papenbrock, et al., Phys. Lett. B 734, 333 (2014)

[42] P. Moskal, J. Smyrski, Acta Phys. Polon. B 41, 2281 (2010)

[43] C. Wilkin, Acta Phys. Pol. B 47, 249 (2016)

[44] Q. Haider, L.-C. Liu, Int. J. Mod. Phys. E 24, 1530009 (2015)

[45] Q. Haider, L.-C. Liu, J. Phys. G 37, 125104 (2010)

[46] B. Krusche, C. Wilkin, Prog. Part. Nucl. Phys. 80, 43 (2014)

[47] M. Skurzok et al., Acta Phys. Polon. B 47, 503 (2016)

[48] P. Moskal, Acta Phys. Polon. B 47, 97 (2016)

[49] P. Moskal, M. Skurzok and W. Krzemien, AIP Conf. Proc. 1753, 030012 (2016)

[50] C. Wilkin, Eur. Phys. J. A 53 no 6, 114 (2017)

[51] P. Moskal, J. Smyrski, Acta Phys. Polon. B 41, 2281 (2010)

[52] W. Krzemien et al., Int. J. Mod. Phys. A 24, 576 (2009)

[53] M. Skurzok et al., EPJ Web of Conferences 181, 01014 (2018)

[54] O. Rundel et al., Acta Phys. Polon. B 48, 1807 (2017)

[55] O. Rundel et al., EPJ Web Conf. 130, 02008 (2016) 